\title{
Applications of light speed expansion and gravitational self-energy density in black hole cosmology
}

\author{
U. V. S. Seshavatharam ${ }^{1 *}$, E. Terry Tatum ${ }^{2}$, S. Lakshminarayana ${ }^{3}$ \\ ${ }^{1}$ Honorary faculty, I-SERVE, Alakapuri, Hyderabad-35,AP, India \\ ${ }^{2} 760$ Campbell Ln. Ste. 106 \#161, Bowling Green, KY 42104 \\ ${ }^{3}$ Dept. of Nuclear Physics, Andhra University, Visakhapatnam-03,AP, India \\ *Corresponding authorE-mail: seshavatharam.uvs@gmail.com
}

\begin{abstract}
From the beginning of Planck scale to the scale of the current Hubble radius: 1) Considering the relation, $\left(G M_{t} / c^{2}\right) \cong R_{t} \cong\left(c / H_{t}\right)$, subjects of black holes and cosmology, both can be integrated into evolving black hole cosmology and cosmic horizon problem can be relinquished. 2) Considering 'continuous light speed expansion' of the cosmic black hole horizon, attributed results of cosmic inflation can be re-addressed completely. If 'nature' of the universe is to expand with light speed, then there is no need to think about the existence of currently believed 'Lambda term'. In addition, 'light speed expanding cosmic space' can be called as 'flat space'. 3) Considering the ratio of gravitational self-energy density and thermal energy density to be $\left[\left(9 H_{t}^{2} c^{2} / 20 \pi G\right) / a T_{t}^{4}\right] \cong\left[1+\ln \left(T_{p l} / T_{t}\right)^{2}\right]^{2}$ (where $T_{p l}$ is the Planck scale temperature, and $T_{t}$ is cosmic temperature at any time). Quantum gravity can be implemented in low energy scale current cosmological observations. Considering the above concepts, currently believed dark matter energy density and visible matter energy density both can be accurately fitted with the ratio of current gravitational self-energy density and current thermal energy density. To proceed further, the authors would like to highlight the following three points: 1) Deep-space red shift non-linearity can be expected to be connected with cosmological gravitational and relativistic effects and cannot be considered as a major criterion of cosmic evolution. 2) Until one finds solid applications of super luminal speeds and super luminal expansions in other areas of physics like astrophysics and nuclear astrophysics, currently believed 'cosmic inflation' cannot be considered as a real physical model and alternative proposals of inflation can be given a chance in exploring the evolving history of the universe. 3) Implementing Planck scale in current paradigm of cosmological observations and standard cosmology is very challenging and is inevitable.
\end{abstract}

Keywords: Black Hole Cosmology; Light Speed Expansion; Gravitational Self Energy Density.

\section{Introduction}

Implementing Planck scale in current paradigm of cosmological observations and standard cosmology is very challenging and is inevitable. It is very clear to say that, from quantum gravity point of view or from Planck scale implementation point of view, currently believed modern cosmology is very weak in many aspects and one must accept the fact that, with currently believed modern cosmological concepts and relations it is impossible to implement Planck scale in current cosmological observations. Most-recent cosmological research data strongly suggests that, current universe is expanding at constant speed and evidence for cosmic acceleration is only marginal (Nielsen JT et al 2015). Mainstream researchers started to suggest that, the universe was expanding at light speed (Jun-Jie Wei et al 2015, Melia F, Maier R.S. 2013). Above points strongly necessitate the need of revising the foundations of accelerating model of cosmology. The authors would like to stress the fact that based on the Planck scale and independent of standard cosmology, with very simple mathematical relations, it is certainly possible to develop a unified model of quantum cosmology that connects currently observed Hubble parameter and cos mic microwave background temperature. The key theme of the authors' published paper (Seshavatharam, U.V.S et al 2015) is to fit the present-day Hubble parameter, dark matter density and visible (baryonic) matter density with the current cosmic microwave background temperature and the Planck scale Hubble parameter. It is currently believed that, in order to explain many observations such as Type Ia supernovae data and baryon acoustic oscillations, the cosmological constant and dark matter must be integrated into the cosmological model. It may also be noted that, in order to obtain the value of the Hubble parameter, cosmologists assume the existence of dark matter and dark energy. Sophisticated statistical methods are required to determine the present day Hubble parameter. This is the case with the recent Planck survey analysis (Planck Collaboration 2015). Independent of this routine procedure and without considering galactic redshift data, a heuristic model of evolving black hole cosmology can be developed with three simple assumptions. This new approach to quantum cosmology connects special theory of relativity, general theory of relativity and the Planck scale at utmost fundamental level.

Progress towards final unification has proceeded by a series of 'successive approximations, allowing more and more accurate 
observations over a wider and wider range of phenomena. In modern cosmology, it is generally believed that it makes no sense to model our universe without assuming the existence of cosmic acceleration or galactic red shifts. In this letter, without incorporating Friedmann concepts and without considering galactic red shifts, the authors developed 'light speed evolving black hole cosmology' in a scale independent quantum gravitational approach and succeeded in fitting the basic observed physical parameters of the current universe with surprising accuracy! During its evolution, as the universe is assumed to be expanding at light speed, it is natural to think about 'formation of galaxies' and 'galactic receding' from and about the cosmic centre in all directions. The authors would like to stress the fact that, 'galactic red shift' cannot be considered as a major criterion of cosmic evolution.

Photons and black holes can be considered as the best candidates of quantum gravitational objects. It is true that, without the existence of the universe, there is no independent existence to any photon or any black hole. Now the fundamental question to be answered is: Is our universe a quantum gravitational object or something else? Physicists expressed several opinions with many possible solutions. Astrophysicists believe that, the universe constitutes so many galaxies, and each galaxy constitutes a massive central black hole. Some of the black hole physicists believe in the existence of primordial black holes also. When the early universe was able to create a number of galactic black holes or primordial black holes, it may not be a big problem for the whole universe to behave like a big primordial evolving black hole. With reference to the current concepts of modern cosmology, probability of 'this' to happen may be zero, but its possibility cannot be ruled out. By considering the current observable universe as an evolving primordial black hole, many fundamental issues of cosmology can be resolved. It may be noted that, when modern cosmology was in its development stage, black hole physics was in its budding stage. In an optimistic approach, some of the modern cosmologists believe that, during cosmic evolution, Planck scale quantum gravitational interactions might have an observable effect on the current observable cosmological phenomena. Clearly speaking, with respect to 'Quantum gravity' and Planck scale early universal laboratory, current universe can be considered as a low-energy scale laboratory. If so, cosmological quantum gravity can be considered as scale independent. If one is willing to consider the current observable universe as a low-energy scale laboratory, currently believed cosmic microwave background temperature can be considered as the low-energy quantum gravitational effect. At any time in the past, i.e as the operating energy scale was assumed to be increasing; past high cosmic background temperature can be considered as the high-energy quantum gravitational effect. Thinking in this way, starting from the Planck scale, quantum cosmology can be considered as 'scale independent model' and the universe can be considered as the best quantum gravitational object.

\section{Three simplified assumptions}

From the Planck scale to the scale of our observable universe, three workable and simple assumptions can be expressed as follows:

Assumption-1: Right from the beginning of the Planck scale, the cosmic horizon is expanding at light speed.

Comment-1: Even though this assumption is ad-hoc, it can be supported by the recently published (arXiv and other main-stream journal) papers $[1,2,3]$ reporting a critical re-evaluation of the Type Ia supernovae data. If 'nature' of the universe is to expand with light speed, then there is no need to think about the existence of currently believed 'Lambda term'. 'Cosmic flatness' can be defined with this assumption. It may be noted that, so far no theoretical model explained the reasons for the photon's light speed. Moreover, currently believed standard physical concepts and current cosmological observations, all are completely based on the 'light speed' concept only. Even though standard cosmology is well standing on 'inflation', there is no clear-cut reasoning for its super luminal speed of expansion and super swelling. As a compromising solution in between 'physics' and 'physical observations, general theory of relativity and special theory of relativity both can be firmly coupled with 'continuous light speed expansion'. Consequences of light speed expansion seem to be practical and fruitful.

Assumption-2: Beginning with the Planck scale, the cosmic radius and Hubble parameter follow the relation,

$$
R_{t} \cong \frac{G M_{t}}{c^{2}} \cong \frac{c}{H_{t}}
$$

Where $R_{t}, M_{t}, H_{t}$ and $c$ represent the radius, mass, Hubble parameter and expansion speed at time $t$ respectively.

Comment-2: For accurate fitting of the current Hubble parameter and the current CMBR temperature, the authors ignored the factor 2 of the Schwarzschild black hole radius formula. This modified assumption appears in many papers describing closed cosmology models. It paves a way to understand the cosmic horizon problem. At every stage of cosmic expansion, universal matter is confined within a radius limited by $\left[R_{t} \cong G M_{t} / c^{2}\right] \cong\left[c / H_{t}\right]$.

Assumption-3: Beginning with the Planck scale, at any stage of cosmic expansion, the ratio of cosmic gravitational potential energy density and thermal energy density is equal to $\left[1+\ln \left(\frac{T_{p l}}{T_{t}}\right)^{2}\right]$ where $T_{p l}$ is the Planck scale temperature and $T_{t}$ is the cosmic temperature at time $\mathrm{t}$.

$$
\left.\frac{\left(\rho_{g s e}\right)_{t} c^{2}}{a T_{t}^{4}} \cong\left[1+\ln \left(\frac{T_{p l}}{T_{t}}\right)^{2}\right]\right\}
$$

Where,

$$
\left(\rho_{g s e}\right)_{t} c^{2} \cong\left[\frac{3}{5} \frac{G M_{t}^{2}}{R_{t}}\right] /\left[\frac{4 \pi}{3}\left(R_{t}^{3}\right)\right]
$$

Here, at any stage of cosmic expansion, $\left[\frac{3}{5} \frac{G M_{t}^{2}}{R_{t}}\right]$ represents the magnitude of cosmic gravitational self-energy and $\frac{4 \pi}{3}\left(R_{t}^{3}\right)$ represents the cosmic Hubble volume. With this idea, current dark matter density and visible matter density both can be fitted accurately. See section 5. Thus,

$$
\left.\begin{array}{l}
{\left[\left(9 c^{4} / 20 \pi G R_{t}^{2}\right) / a T_{t}^{4}\right] \cong\left[1+\ln \left(T_{p l} / T_{t}\right)^{2}\right]^{2} \text { and }} \\
{\left[\left(9 H_{t}^{2} c^{2} / 20 \pi G\right) / a T_{t}^{4}\right] \cong\left[1+\ln \left(T_{p l} / T_{t}\right)^{2}\right]^{2}}
\end{array}\right\}
$$

Comment-3: This assumption is new and can be given some consideration for in-depth analysis. This model assumes that, at the Planck scale, cosmic gravitational self-energy density and thermal energy density are equal in magnitude. During subsequent cosmic evolution, gravitational self-energy density is always higher than the thermal energy density by the authors' proposed scaling factor, It may be noted that, even though the current universe is expanding at light speed,

1) Current universe is outsized;

2) Current Hubble parameter is very small;

3) Current rate of Hubble parameter decline is very small; 
4) Assumed to follow the relation (5), i.e.

$$
\left(9 H_{0}^{2} c^{2} / 20 \pi G\right) \cong\left[1+\ln \left(T_{p l} / T_{0}\right)^{2}\right]^{2} a T_{0}^{4}
$$

Based on these four points, the decline in current thermal energy density must be very minute and may reflect an "isotropic" behavior. In reality, as the universe is always assumed to be expanding at 'speed of light', there is no scope for 'temperature isotropy', and cosmic temperature will always tends to decrease. Since the current observable universe is very large and as the observer is not in a position to reach all parts of the current universe, one may be forced to arrive at a misconception of 'CMBR isotropy'.

\section{To connect the cosmic physical parameters}

Following these assumptions, the Planck scale Hubble parameter can be expressed as follows:

$$
H_{p l} \cong \frac{c^{3}}{G M_{p l}} \cong \frac{c}{R_{p l}} \cong 1.85492 \times 10^{43} \mathrm{sec}^{-1}
$$

Where $R_{p l} \cong G M_{p l} / c^{2} \cong \sqrt{G \hbar / c^{3}} \cong 1.6162 \times 10^{-35} \mathrm{~m}$ is the Planck length and the assumed radius connected with the Planck mass. Planck scale cosmic temperature can be expressed as

$$
T_{p l} \cong\left(\frac{9 H_{p l}^{2} c^{2}}{20 \pi G a}\right)^{1 / 4} \cong 9.67791 \times 10^{31} \mathrm{~K}
$$

At any stage of cosmic expansion, cosmic temperature can be related by

$$
\begin{aligned}
& H_{t} \cong\left[1+\ln \left(T_{p l} / T_{t}\right)^{2}\right] \sqrt{\frac{20 \pi G a T_{t}^{4}}{9 c^{2}}} \\
& \left.\cong\left\{\left[1+\ln \left(T_{p l} / T_{t}\right)^{2}\right]\left(T_{t} / T_{p l}\right)^{2}\right\} H_{p l}\right\} \\
& R_{t} \cong\left[1+\ln \left(T_{p l} / T_{t}\right)^{2}\right]^{-1} \sqrt{\frac{9 c^{4}}{20 \pi G a T_{t}^{4}}} \\
& \cong\left\{\frac{\left(T_{p l} / T_{t}\right)^{2}}{\left[1+\ln \left(T_{p l} / T_{t}\right)^{2}\right]}\right\} R_{p l}
\end{aligned}
$$

\section{Current cosmic physical parameters}

As per the 2015 Planck data (Planck Collaboration 2015), the current value of the Hubble parameter is reported to be:

Planck TT+low P: $(67.31 \pm 0.96) \mathrm{km} / \mathrm{sec} / \mathrm{Mpc}$

Planck TE+low P: $(67.73 \pm 0.92) \mathrm{km} / \mathrm{sec} / \mathrm{Mpc}$

Planck TT,TE,EE+low P: $(67.7 \pm 0.66) \mathrm{km} / \mathrm{sec} / \mathrm{Mpc}$

And the current value of CMBR temperature is:

$\left.\begin{array}{l}\text { Planck TT + lowP + BAO: }(2.722 \pm 0.027) \mathrm{K} \\ \text { Planck TT; TE; EE + low P + BAO: }(2.718 \pm 0.021) \mathrm{K}\end{array}\right\}$

In this paper, for calculation purpose, the authors consider

$T_{0} \cong 2.722 \mathrm{~K}$.

Step-1: Current cosmic radius can be estimated as follows:
$R_{0} \cong\left[1+\ln \left(T_{p l} / T_{0}\right)^{2}\right]^{-1} \sqrt{\frac{9 c^{4}}{20 \pi G a T_{0}^{4}}}$
$\cong 1.396522 \times 10^{26} \mathrm{~m} \cong 14.76$ Billion Ly

Step-2: Current Hubble parameter can be estimated as follows:

$$
\begin{aligned}
& H_{0} \cong\left[1+\ln \left(T_{p l} / T_{0}\right)^{2}\right] \sqrt{\frac{20 \pi G a T_{0}^{4}}{9 c^{2}}} \\
& \cong 2.14671 \times 10^{-18} 1 / \mathrm{sec} \cong 66.241 \mathrm{~km} / \mathrm{sec} / \mathrm{Mpc} .
\end{aligned}
$$

Step-3: Current cosmic mass can be estimated as,

$$
M_{0} \cong \frac{R_{0} c^{2}}{G} \cong \frac{c^{3}}{G H_{0}} \cong 1.88067 \times 10^{53} \mathrm{~kg}
$$

Total estimated mass of our current universe is approximately 1.46 $\mathrm{x} 10^{53} \mathrm{~kg}$. This can be compared with above estimate of $1.88 \mathrm{x}$ $10^{53} \mathrm{~kg}$. Estimation of visible baryonic mass mainly depends on estimating the number of galaxies, estimating the number of stars per galaxy and estimating the average mass per star. This entire procedure is based on observation and requires many correction factors!

\section{Predicting and fitting dark matter and visi- ble matter energy densities}

If one is willing to consider the 'Planck mass' as a real matter particle, then close to the Planck scale, one can expect visible matter content as well as dark matter content. With this idea, in this section, by considering the ratio of gravitational self-energy density and thermal energy density, in a cosmological approach, the authors made an attempt to fit the present dark matter energy density and visible matter energy density accurately. Assuming that, at the Planck scale, the magnitude of gravitational self-energy is equal to the sum of the magnitude of dark matter energy and the magnitude of visible matter energy, value of $k$ can be defined as $k \cong 5.7805$. Its assumed background relation connected with the Planck scale can be expressed as follows:

$$
\begin{aligned}
& \left(e^{x_{p l}}-1\right)+\left(e^{x_{p l}^{2}}-1\right) \cong 1 \\
& \rightarrow e^{x_{p l}}+e^{x_{p l}^{2}} \cong 3 \text { and }
\end{aligned}
$$

By trial-error method, $x_{p l} \cong 0.522425$

$$
\Rightarrow k \cong \exp \left(\frac{1}{x_{p l}}\right)-1 \cong 5.7805
$$

With reference to the currently believed data connected with dark matter and visible matter energy densities, it is possible to guess that,

$$
\begin{aligned}
& \left(\rho_{d}\right)_{t} c^{2} \cong\left(\mathrm{e}^{x_{t}}-1\right)\left(\frac{9 H_{t}^{2} c^{2}}{20 \pi G}\right) \\
& \text { where, } \left.x_{t} \cong\left\{\ln \left(k+\sqrt{\frac{\left(9 H_{t}^{2} c^{2} / 20 \pi G\right)}{a T_{t}^{4}}}\right)\right\}^{-1}\right\}
\end{aligned}
$$

Where $\left(\rho_{d}\right)_{t} c^{2}$ is the dark matter energy density at time $t$. Current dark matter energy density (Planck Collaboration 2015) can be fitted as follows: 


$$
\left.\begin{array}{l}
\left(\rho_{d}\right)_{0} c^{2} \cong\left(\mathrm{e}^{x}{ }^{-1}\right)\left(\frac{9 H_{0}^{2} c^{2}}{20 \pi G}\right) \\
\cong 1.958 \times 10^{-10} \mathrm{~J} \cdot \mathrm{m}^{-3} \cong 2.17857 \times 10^{-27} \mathrm{~kg} \cdot \mathrm{m}^{-3} \\
\cong 0.2643 *\left(\frac{3 H_{0}^{2} c^{2}}{8 \pi G}\right) \\
\text { where, } x_{0} \cong\left\{\ln \left(k+\sqrt{\frac{\left(9 H_{0}^{2} c^{2} / 20 \pi G\right)}{a T_{0}^{4}}}\right)\right\}^{-1}
\end{array}\right\}
$$

Similarly, it is possible to guess that,

$$
\begin{aligned}
& \left(\rho_{m}\right)_{t} c^{2} \cong\left(\mathrm{e}^{y_{t}}-1\right)\left(\frac{9 H_{t}^{2} c^{2}}{20 \pi G}\right) \\
& \text { where, } y_{t} \cong\left\{\ln \left(k+\sqrt{\frac{\left(9 H_{t}^{2} c^{2} / 20 \pi G\right)}{a T_{t}^{4}}}\right)\right\}^{-2} \cong x_{t}^{2}
\end{aligned}
$$

Where $\left(\rho_{m}\right)_{t} c^{2}$ is the visible (baryonic) matter energy density at time $t$. Current visible matter energy density can be fitted as follows:

$$
\begin{aligned}
& \left(\rho_{m}\right)_{0} c^{2} \cong\left(\mathrm{e}^{y_{0}}-1\right)\left(\frac{9 H_{0}^{2} c^{2}}{20 \pi G}\right) \\
& \cong 3.592 \times 10^{-11} \mathrm{~J} \cdot \mathrm{m}^{-3} \cong 3.997 \times 10^{-28} \mathrm{~kg} \cdot \mathrm{m}^{-3} \\
& \cong 0.0485 *\left(\frac{3 H_{0}^{2} c^{2}}{8 \pi G}\right) \\
& \text { where, } y_{0} \cong\left\{\ln \left(k+\sqrt{\frac{\left(9 H_{0}^{2} c^{2} / 20 \pi G\right)}{a T_{0}^{4}}}\right)\right\}^{-2} \cong x_{0}^{2}
\end{aligned}
$$

\section{Cosmic age}

In general, cosmic age estimates are model-dependent and cosmic size-dependent. In this proposed model, cosmic age estimation is very simple and direct. As the cosmic model is always assumed to be expanding with light speed, from the beginning of Planck scale, cosmic age can be estimated as follows:

$$
\left.\begin{array}{l}
t \cong \frac{\left(R_{t}-R_{p l}\right)}{c} \text { and } \\
c t \cong\left(R_{t}-R_{p l}\right) \simeq R_{t}
\end{array}\right\}
$$

For the current case, since $\left(R_{0} \square R_{p l}\right)$ and $\left(R_{0}-R_{p l}\right) \cong R_{0}$.

$$
t_{0} \cong \frac{R_{0}}{c} \cong \frac{1}{H_{0}} \cong 14.76 \text { Billion Years }
$$

\section{Model equations of cosmic non-liner red- shift}

During its evolution, as universe is assumed to be expanding at light speed, it is natural to think about 'formation of galaxies' and 'galactic receding' from and about the cosmic centre in all directions. The authors would like to stress the following two points.

a) Galactic redshift cannot be considered as a major criterion of cosmic evolution.

b) Clearly speaking, observed galactic red shift is a consequence of cosmic evolution and not a deciding factor of current and future cosmic expansion.

In this section, in a semi-empirical approach, the authors propose four (published) model equations of non-linear galactic red shift (Tatum, E.T et al, 2015a, 2015b).

Model equation-1:

$$
\begin{aligned}
& Z \cong \sqrt{\frac{R_{0}}{R_{t}}-1} \cong \sqrt{\frac{G M_{0}}{c^{2} R_{t}}-1} \\
& \text { where } R_{t}<R_{0} \text {, and } M_{0} \cong c^{3} / G H_{0}
\end{aligned}
$$

With reference to cosmic gravitational self-energy, dark matte energy and visible matter energy, (and neglecting total thermal energy), leftover energy can be expressed as follows.

If $x_{t} \cong\left\{\ln \left(k+\sqrt{\frac{\left(9 H_{t}^{2} c^{2} / 20 \pi G\right)}{a T_{t}^{4}}}\right)\right\}^{-1}$,

At any stage of cosmic evolution, approximately

$$
\begin{aligned}
& \text { Left over energy } \cong\left(E_{l o}\right)_{t} \\
& \cong\left[3-\left(e^{x_{t}}+e^{x_{t}^{2}}\right)\right]\left(\frac{9 H_{t}^{2} c^{2}}{20 \pi G}\right)\left[\frac{4 \pi}{3}\left(\frac{c}{H_{t}}\right)^{3}\right]
\end{aligned}
$$

If one is willing to call this unaccountable energy as 'invisible energy' - it can be called as 'dark energy' or 'vacuum energy'. If one is willing to consider this unaccountable energy as the source
Where $R_{0}$ and $R_{t}$ represent current and past cosmic radii, respectively, pertaining to specific astronomical observations.

By following Minkowski's flat space relativistic Doppler shift formula, calculated galactic and CMBR redshifts can be tightly correlated with observations. With respect to the proposed assumptions it is clear that at any stage of cosmic expansion, cosmic radius is approximately inversely proportional to the squared cosmic temperature. The above relation (21) can be expressed as follows.

$$
Z \cong \sqrt{\frac{R_{0}}{R_{t}}-1} \cong \sqrt{\frac{T_{t}^{2}}{T_{0}^{2}}-1}
$$


Where $T_{t}$ is the past cosmic temperature and $T_{0}$ is the current cosmic temperature and $T_{t}>T_{0}$. For past higher cosmic temperatures, where $T_{t} \gg T_{0}$

$$
Z \cong \sqrt{\frac{T_{t}^{2}}{T_{0}^{2}}-1} \cong \frac{T_{t}}{T_{0}}
$$

Model equations-2 and 3:

With reference to light speed expansion, Minkowski's relativistic Doppler formula can also be given some consideration:

$$
Z+1 \cong \sqrt{\frac{[1+(v / c)]}{[1-(v / c)]}}
$$

The velocity term $v$ in this Minkowski formula can be substituted with $\left[1-\left(R_{x} / R_{0}\right)\right] c$ where $R_{x}<R_{0}$. the reduced relation can be expressed as follows.

$$
Z \cong \sqrt{\frac{2-\left(R_{x} / R_{0}\right)}{\left(R_{x} / R_{0}\right)}}-1
$$

Model equation-4:

Another interesting relation can be expressed as follows.

$$
Z \cong\left\{\left(\frac{\rho_{t}}{\rho_{0}}\right)^{\frac{1}{3}} \ln \left[1+\left(\frac{\rho_{t}}{\rho_{0}}\right)^{\frac{1}{3}}\right]^{-1}\right\}-\frac{1}{\ln (2)}
$$

Where $\rho_{t} \cong\left(3 H_{t}^{2} / 8 \pi G\right)$ and $\rho_{0} \cong\left(3 H_{0}^{2} / 8 \pi G\right)$ respectively. Important point to be noted is that, currently believed CMBR redshift of 1100 and $3000 \mathrm{~K}$ related with first hydrogen atom can be fitted easily. These proposed and published relations (21) to (26) can be recommended for in-depth analysis at fundamental level.

\section{Discussion}

Until one finds solid applications of super luminal speeds and super luminal expansions in other areas of physics like astrophysics and nuclear astrophysics, currently believed 'cosmic inflation' cannot be considered as a real physical model and alternative proposals of inflation can be given a chance in exploring the evolving history of the universe. Good qualities of any new model are: 1) Good motivation and in-depth insight; 2) Strong reasoning; 3) Unified approach and conceptual simplicity; 4) Accuracy in data fitting; 5) more applications; 6) Eliminating the ambiguity in current models. The authors are sure that, this proposed model covers all these points at an utmost fundamental level by at least $60 \%$ and request the science community to review this model in a true scientific spirit. With three simple assumptions and without considering cosmic acceleration or red shift concepts, proposed 'light speed evolving black hole cosmology' model succeeded in fitting the basic observed physical parameters of the current universe with surprising accuracy!

\subsection{About Assumption-1}

In this proposed model cosmic horizon is assumed to be expanding at light speed. This assumption seems to be strongly supported by recent papers $[1,2,3]$ presenting a critical analysis of the Type Ia supernovae data which suggests that the evidence of cosmic acceleration is marginal at best, and that our universe may well be expanding at a constant speed. This conclusion is especially wellsupported in references. It should be noted that, 'expansion at constant speed' implies that no apparent net forces are acting on the expanding universal system as a whole. In addition, if the very nature of universe is to expand with light speed, then there is no need to think about the existence of currently believed 'Lambda term'.

In this proposed model, within the first second of cosmic expansion, the universe expands from $1.6162 \times 10^{-35} \mathrm{~m}$ to $3 \times 10^{8} \mathrm{~m}$ and the ratio of expansion is $1.85 \times 10^{43}$. Similarly, in one second from the Planck scale, temperature drops from $9.68 \times 10^{31} \mathrm{~K}$ to $2.873 \times 10^{10} \mathrm{~K}$ and the ratio of temperature drop in the first second is $3.37 \times 10^{21}$. Thinking in this way, the early and modern concepts of inflation (Guth AH. 1981) can be understood.

\subsection{About assumptions 2 and 3}

Here the authors would like to stress the following facts:

a) Considering assumption-2, the cosmic horizon problem can be relinquished. At any stage of cosmic expansion, no matter can exist beyond the cosmic radius, as defined by $\left[R_{t} \cong G M_{t} / c^{2}\right] \cong\left[c / H_{t}\right]$.

b) Considering assumptions 2 and 3 together, current CMBR temperature, Hubble parameter, dark matter energy density and visible matter density can be fitted accurately.

c) With reference to data-fitting, the authors' proposed method is completely new, simple and surprisingly accurate. At any stage of cosmic expansion, the characteristic expression $\ln \left(\sqrt{\left(9 H_{t}^{2} c^{2} / 20 \pi G\right) / a T_{t}^{4}}\right)$ might be considered as a useful index of dark matter percentage and visible matter percentage and can be recommended for further study. .

\section{Conclusion}

Progress towards final unification has proceeded by a series of 'successive approximations', allowing more and more accurate observations over a wider and wider range of phenomena. Day by day, 'accelerating model of cosmology' is losing its basic identity. In addition, 'dark energy' and 'Lambda term' both seem to remain as virtual objects of modren cosmology. Anyhow, from unification point of it is a must to implemet Planck scale in understanding early cosmological predictions and current cosmological observations. In this context, the authors would like to stress the fact that, by introducing cosmic 'light speed expansion', 'Schwarzschild radius' and 'gravitational self-energy density', a potentially useful 'evolving black hole cosmology' can be developed. The basic advantages of this model can be expressed in the following way:

a) Planck scale can be successfully implemented in understanding past and current cosmological predictions and observations.

b) Hubble parameter and cosmic temperature can be inter-linked at fundamenatl level.

c) Observed isotropic nature of CMBR can be understood well. It may be noted that, as the universe is always assumed to be expanding at 'speed of light', there is no scope for 'temperature isotropy' and cosmic temperature will always tends to decrease. Since the current observable universe is very large and as the observer is not in a position to reach all parts of the current universe, one may be forced to arrive at a misconception of 'CMBR isotropy'.

d) Time to time, visible matter energy density and dark matter energy desnity can be predicted and thereby their creation rate can be understood.

e) Attributed results of currently believed 'cosmic inflation' can be understood well with continuous light speed expansion.

f) Cosmic hoizon problem can be relinquished at fundamental level.

g) Deep space galactic redshift can be understood as a consequence of cosmological gravitational effect and cannot 
be considered as a deciding factor of current and future cosmic expansion

h) Special theory of relativity, General theory of relativity and Quantim mechanics can be studied and a unified model of quantum cosmology can be developed.

\section{Acknowledgements}

One of the authors, Seshavatharam U.V.S, is indebted to professors K.V. Krishna Murthy, Chairman, Institute of Scientific Research in Vedas (I-SERVE), Hyderabad, India and Shri K.V.R.S. Murthy, former scientist IICT (CSIR), Govt. of India, Director, Research and Development, I-SERVE, for their valuable guidance and great support in developing this subject.

\section{References}

[1] Nielsen JT, et al. Marginal evidence for cosmic acceleration from Type IA supernovae. ArXiv: 1506.01354v2; (2015).

[2] Jun-Jie Wei et al. A comparative analysis of the supernova legacy survey sample with $\Lambda \mathrm{CDM}$ and the $\mathrm{R}_{\mathrm{h}}=\mathrm{ct}$ Universe. The Astronomical Journal. 149:102. (2015) http://dx.doi.org/10.1088/0004$6256 / 149 / 3 / 102$.

[3] Melia F, Maier RS. Cosmic chronometers in the $\mathrm{R}_{\mathrm{h}}=\mathrm{ct}$ Universe Mon. Not. Roy. Astron. Soc.; 432:2669. (2013) http://dx.doi.org/10.1093/mnras/stt596.

[4] Seshavatharam, U.V.S, Tatum, E.T and Lakshminarayana, S. On the role of gravitational self-energy density in spherical flat space quantum cosmology. Journal of Applied Physical Science International. Vol.: 4, Issue. 4, 228-236 (2015).

[5] Planck Collaboration: Planck 2015 Results. XIII. Cosmological Parameters. (2015).

[6] Tatum, E.T., Seshavatharam, U.V.S. and Lakshminarayana, S. The Basics of Flat Space Cosmology. International Journal of Astronomy $\begin{array}{lllll}\text { and Astrophysics, } & 5, & 116-124 & (2015 & \text { a) }\end{array}$ http://dx.doi.org/10.4236/ijaa.2015.52015.

[7] Tatum ET, Seshavatharam U.V.S, Lakshminarayana S. Flat space cosmology as a mathematical model of quantum gravity or quantum cosmology. International journal of astronomy and astrophysics. 5, 133-140. (2015b).

[8] Guth AH. Inflationary universe: A possible solution to the horizon and flatness problems. Phys. Rev.; D23:347. (1981) http://dx.doi.org/10.1103/PhysRevD.23.347. 